NOTE

\title{
Mitogenome analysis reveals a complex phylogeographic relationship within the wild tiger population of Thailand
}

\author{
Waradee Buddhakosai ${ }^{1}$, Worata Klinsawat ${ }^{2}$, Olutolani Smith ${ }^{3,4}$, \\ Manakorn Sukmak ${ }^{5}$, Nongnid Kaolim ${ }^{6}$, Somphot Duangchantrasiri ${ }^{7}$, \\ Achara Simcharoen ${ }^{7}$, Boripat Siriaroonrat ${ }^{8}$, Worawidh Wajjwalku ${ }^{1,6, *}$
}

${ }^{1}$ Interdisciplinary Graduate Program in Genetic Engineering, Graduate School, Kasetsart University, Bangkok 10900, Thailand ${ }^{2}$ Department of Fisheries, Wildlife and Conservation Biology, University of Minnesota, St. Paul, MN 55108, USA ${ }^{3}$ Division of Biology, Silwood Park, Imperial College London, Ascot, Berks SL5 7PY, UK ${ }^{4}$ Panthera, 8 West 40th St, 18th Floor, New York, NY 10018, USA

${ }^{5}$ Department of Farm Resources and Production Medicine, Faculty of Veterinary Medicine, Kasetsart University, Thailand ${ }^{6}$ Department of Pathology, Faculty of Veterinary Medicine, Kasetsart University, Kamphaengsaen, Nakhon Pathom 73140 , Thailand

${ }^{7}$ Department of National Parks, Wildlife and Plant Conservation Department, Bangkok 10900, Thailand

${ }^{8}$ Zoological Park Organization, Dusit, Bangkok 10300,Thailand

\begin{abstract}
We present the first study of the complete mitogenome of wild tigers Panthera tigris in Thailand. Thailand has been recognised as one of the most important countries within the geographic range of $P$. tigris and is home to 2 subspecies: Indochinese $P$. $t$. corbetti in the north and Malayan P. t. jacksoni in the south. We obtained samples from wild tigers in a large forest complex in northern Thailand (Western Forest Complex, WEFCOM) and from locally confiscated individuals in southern Thailand close to the border with Malaysia. Our results support the occurrence of both Indochinese and Malayan tigers in Thailand and reveal complex phylogeographic patterns of the wild tiger population in Southeast Asia.
\end{abstract}

KEY WORDS: mtDNA - Panthera tigris corbetti - Panthera tigris jacksoni - Western Forest Complex $\cdot$ WEFCOM $\cdot$ Large cats $\cdot$ Phylogenetics

\section{INTRODUCTION}

Morphological and genetic studies of wild tigers Panthera tigris have resulted in the designation of 6 extant subspecies: Amur P. t. altaica, Bengal P. t. tigris, South China P. t. amoyensis, Sumatran P. t. sumatrae, Indochinese P. t. corbetti and Malayan P. t. jacksoni (Maźak 1981, Luo et al. 2004). Thailand is one of 7 countries situated within the geographic

\footnotetext{
*Corresponding author: fvetwww@yahoo.com
}

range of tigers and contains sufficient habitat to support 2 source sites and up to 250 tigers (DNP 2010, Walston et al. 2010). The majority of tigers in Thailand are of the subspecies P. t. corbetti, and P. t. jacksoni occurs in the south of the country separated by the Isthmus of Kra (Luo et al. 2004). Most of the remaining tiger habitat in Thailand is within the Western Forest Complex (WEFCOM), a World Heritage Site which covers an area of approximately

() The authors 2016. Open Access under Creative Commons by Attribution Licence. Use, distribution and reproduction are unrestricted. Authors and original publication must be credited. 
$18000 \mathrm{~km}^{2}$ (Simcharoen et al. 2007). However, despite government protection under the Wild Animal Reservation and Protection Act B.E.2535 (1992) of Thailand, the tiger population here is still declining due to habitat loss and human persecution.

Due to the critical importance of regions such as WEFCOM to tiger conservation in Thailand, National Park staff have been monitoring the population through regular camera trap studies, and they have also begun to collect non-invasive samples (faeces and hair) for genetic analysis. Recent genetic studies of mitochondrial DNA variation in the WEFCOM population and in southern Thailand (unpubl. data) have revealed the presence of 2 new haplotypes that have not been previously reported. One haplotype (GenBank Accession No. KC879297) was discovered in tiger samples from WEFCOM, and the other (KC879296), which was detected in an individual from southern Thailand, represents a new haplotype of P. t. jacksoni.

Here we describe a new study as an extension of this work, to characterize the complete mitogenome of tigers from WEFCOM and southern Thailand, and to demonstrate the phylogenetic relationship between these haplotypes and tigers in other populations. The results of this and subsequent genetic studies will act as the basis for a national genetic database, which could be used for both research and forensic applications in Thailand.

\section{MATERIALS AND METHODS}

\section{Primer design}

Most of the primers used in this study were newly designed from the alignment of cymt (GenBank DQ151150) and F2 numt sequences (GenBank DQ151151) (Kim et al. 2006). Primers originally developed for Ursidae (Delisle \& Strobeck 2002) were used to amplify the region between the 2 ends of $F 2$ numt (ATP6 - ND5). Full details of the primers used are given in Table $\mathrm{S} 1$ in the Supplement, at www.intres.com/articles/suppl/n030p125_supp.pdf.

\section{Samples and DNA extraction}

Hair samples $(n=3)$ were collected from WEFCOM during field surveys by National Park staff in 2009. These 3 samples shared the same haplotype based on aligned sequences from ND6, Cyt $b$ and the control region; we therefore chose only 1 sample for further analysis (ID = WEFCOM). Three muscle samples were also obtained from 3 tiger carcasses confiscated by police from poachers in Narathiwat province, southern Thailand, in 2009. One sample was obtained from each individual, and 1 sample (ID = Ti2) was identified as a new haplotype using the aligned sequences from ND6, Cyt $b$ and the control region. This sample was therefore chosen for subsequent analysis. A map of Thailand showing the sampling sites is shown in Fig. S1 in the Supplement at www.int-res. com/articles/suppl/n030p125_supp.pdf.

DNA extractions were performed following a modified method of Carter \& Milton (1993). Briefly, the hair or muscle was cut into small pieces, digested in lysis buffer, and incubated overnight at $56^{\circ} \mathrm{C}$. The supernatant was then collected and mixed with silica diatom. After shaking and centrifugation, the resultant pellet was washed with washing buffer. The pellet was then dried and DNA eluted with elution buffer.

\section{PCR conditions}

Each DNA template was amplified with a singledirection PCR prior to specific fragment amplification. PCRs were performed in a $20 \mu \mathrm{l}$ reaction volume containing $10 \mu \mathrm{l}$ of $2 \times$ Phusion buffer (Finnzymes), $200 \mu \mathrm{M}$ dNTP, $0.25 \mu \mathrm{l}$ of each $1 \mu \mathrm{M}$ reverse primer, $0.2 \mu \mathrm{l}$ of Phusion High-fidelity DNA polymerase (Finnzymes), $2 \mu \mathrm{l}$ of DNA template (diluted to $5 \mathrm{ng}$ $\mathrm{\mu l}^{-1}$ ), and the relevant amount of purified water. PCR cycling conditions were as follows: a pre-denaturation step at $98^{\circ} \mathrm{C}$ for $3 \mathrm{~min}$, then 35 cycles of $98^{\circ} \mathrm{C}$ for $30 \mathrm{~s}$, annealing at $58^{\circ} \mathrm{C}$ for $30 \mathrm{~s}$, extension at $72^{\circ} \mathrm{C}$ for $2 \mathrm{~min}$, then $72^{\circ} \mathrm{C}$ for $5 \mathrm{~min}$. The higher denaturation temperature of $98^{\circ} \mathrm{C}$ is recommended for this highfidelity Taq enzyme.

The second round of PCR was performed in $50 \mu \mathrm{l}$ volumes containing $25 \mu \mathrm{l}$ of $2 \times$ buffer, $200 \mu \mathrm{M}$ dNTP, $0.1 \mu \mathrm{l}$ of each of $10 \mu \mathrm{M}$ forward and reverse primer, $0.5 \mu \mathrm{l}$ of Phusion High-fidelity DNA polymerase (Finnzymes), $1 \mu \mathrm{l}$ of the PCR product from the first round of single-direction PCR and purified water. PCR was performed for 40 cycles under similar conditions to those described above, apart from reducing the extension time to $1 \mathrm{~min}$. PCR products were purified using the silica diatom method as described above (Carter \& Milton 1993) and sequenced in either forward or reverse directions. This 2-step PCR protocol was repeated twice for each mitochondrial region except the control region, which was repeated 3 times. 


\section{Data analysis}

Amplified mtDNA fragments were assembled into complete mtDNA sequences using BioEdit (Hall 1999). Haplotypes identified in this study were then analysed jointly with haplotypes of $4078 \mathrm{bp}$ of concatenated sequence from extant tiger subspecies (Luo et al. 2004); see Fig. 1. Three felid species related to tigers, viz. snow leopard Panthera uncia (GenBank NC_010638), leopard P. pardus (GenBank EF551002) and clouded leopard Neofelis nebulosa (GenBank DQ257669), were used as outgroups in the analysis (Table S2 in the Supplement). Phylogenetic relationships between the 2 tiger samples in this study and published $P$. tigris haplotypes were assessed using neighbourjoining (NJ), maximum parsimony (MP), maximum likelihood (ML) and Bayesian inference. The Bayesian information criterion implemented in jModelTest v2.1.4 (Posada 2008) was used to select the best-fit substitution model for ML and Bayesian analysis. The Hasegawa-Kishino-Yano model + gamma distribution (base $=\left[\begin{array}{llll}0.3250 & 0.2911 & 0.1338\end{array}\right]$, nst $=2$, tratio $=20.6989$, rates $=$ gamma, shape $=$ 0.1520 , ncat $=4$, pinvar $=0$ ) was selected as the best-fit model. Using these model parameters, Bayesian inference was executed in MrBayes v3.2.5 (Ronquist \& Huelsenbeck 2003, Ronquist et al. 2012) for 2000000 generations with trees sampled every 100 generations and the first $25 \%$ generations discarded as burn-in. NJ, MP and ML analyses were performed in PAUP* v4a142 (Swofford 2002) with 2000 repetitions for MP and NJ, and 100 repetitions for ML. Heuristic search with random additions of taxa and tree-bisection-reconnection branch swapping was employed. Trees were then viewed and manually annotated with FigTree v1.4.2. The median-joining phylogenetic network was computed using Network 4.6.1.2 (Bandelt et al. 1999) to analyse the relationship between tiger haplotypes from this study and those from Luo et al. (2004).

Longer sequences of $10140 \mathrm{bp}$ (excluding the control region and the fragment between positions 7399 and 12707) were also evaluated following alignment of complete mtDNA sequences from Zhang et al. (2011), Kitpipit et al. (2012) and Sun et al. (2015). The Tamura-Nei model + gamma distribution (base $=[0.3243,0.2612,0.1509] \mathrm{nst}=6$, rates = gamma, shape $=0.1280$, ncat $=4$, pinvar $=0$ ) was the optimal model. A similar method to that described above was used to construct the tree (see Fig. 2).

\section{RESULTS}

The size of the complete mitogenome in the WEFCOM and Ti2 individuals (GenBank KJ508412 and KJ508413) was 16954 and 17008 bp, respectively (Table S3 in the Supplement). The difference in mitogenome size between this and other studies was mainly due to variation in the tandem repeats TACACACG and TATACACG in the repetitive sequence box 3 of the control region (Fig. S2 in the Supplement). Thirty variable sites were identified in the whole mitogenome excluding the control region (21 C/T and $9 \mathrm{~A} / \mathrm{G}$ nucleotide substitutions), and there were 22 new variable sites (11 in WEFCOM and 11 in Ti2) when comparing our results to other studies (Luo et al. 2004, Driscoll et al. 2009, Zhang et al. 2011, Kitpipit et al. 2012, Mondol et al. 2013, Sun et al. 2015, Xue et al. 2015) (see Table S4 in the Supplement). The WEFCOM haplotype carried 1 Panthera tigris altaica-specific single nucleotide polymorphism (SNP; 14711A) as described by Luo et al. (2004), whereas Ti2 contained no subspecies-specific SNPs.

In each sample, we found 2 distinct sequences with numt contamination in the region of COX2-ATP6. No heteroplasmy was found in either sequence. The first sequence (amplicon 9.1) generated from primers described for Ursidae (Delisle \& Strobeck 2002) contained an F2 numt sequence from the COX1 to COX2 gene as found in the report by Zhang et al. (2011) and could not be assembled with the flanking fragments. The second sequence (amplicon 9.2), amplified by the newly designed primers in this study, contained F2 numt contamination in the COX2 to ATP8 genes, similar to the results of Kitpipit et al. (2012) (see Table S1 and Figs. S3 \& S4 in the Supplement), and perfectly assembled to the adjacent fragments. The last domain of sequences covering part of the ATP6 gene also differed (Figs. S3 \& S4). We therefore excluded the mitochondrial fragment between positions 7399 and 12707 (based on the WEFCOM mitogenome) from further analysis as it may have contained additional numt sequences.

Results from the MP, NJ and ML trees and the Bayesian inference analyses on 4078 bp of concatenated mtDNA indicate that the WEFCOM haplotype is nearly equidistant to the fixed haplotype of P. t. altaica (8 different nucleotides) and a haplogroup consisting of all reported P. t. corbetti mtDNA haplotypes (9 different nucleotides; Fig. 1A). The high Bayesian posterior probability and bootstrap support of this monophyletic cluster suggests a recent common ancestor of tigers now distributed in 


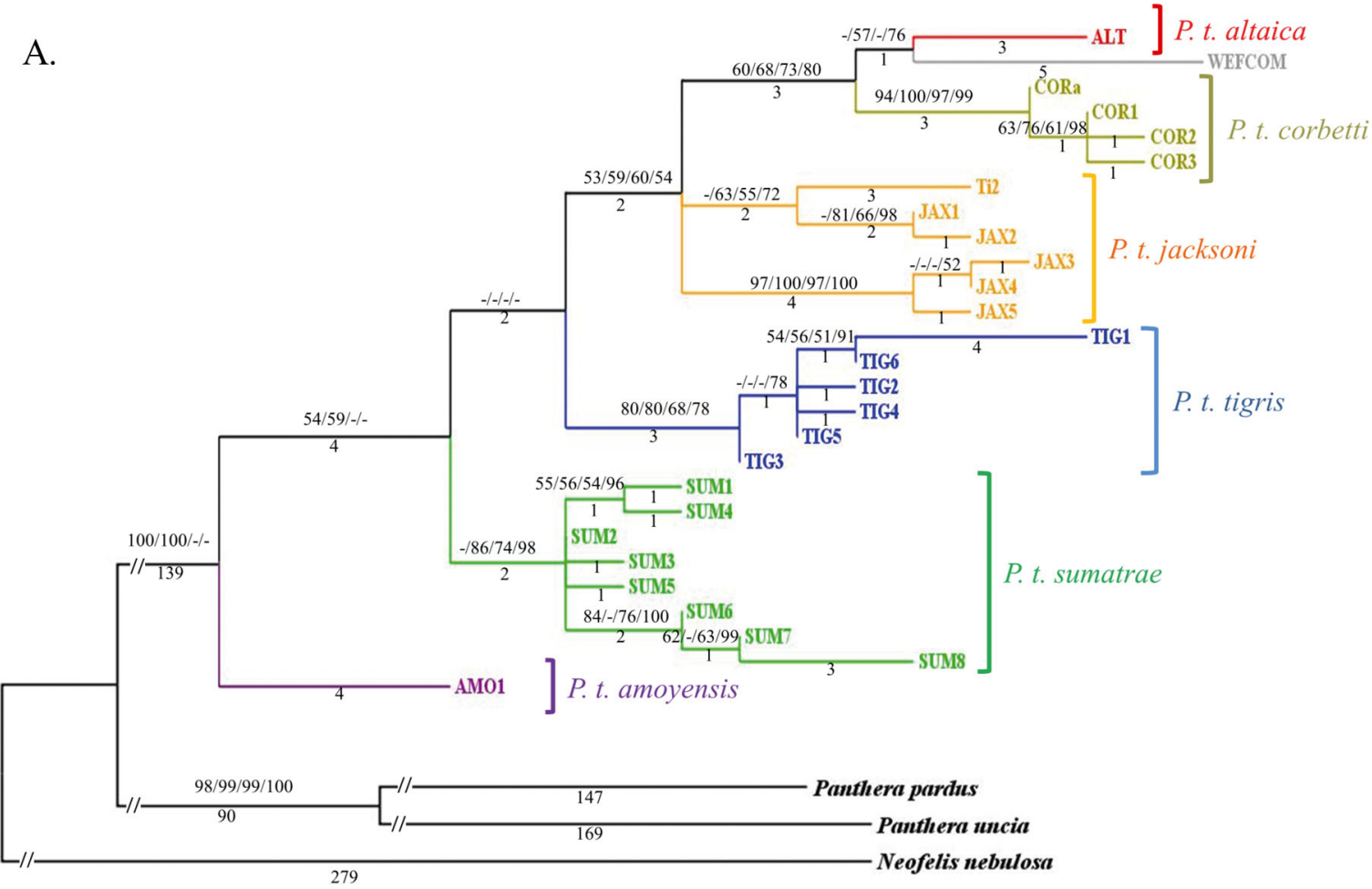

B.

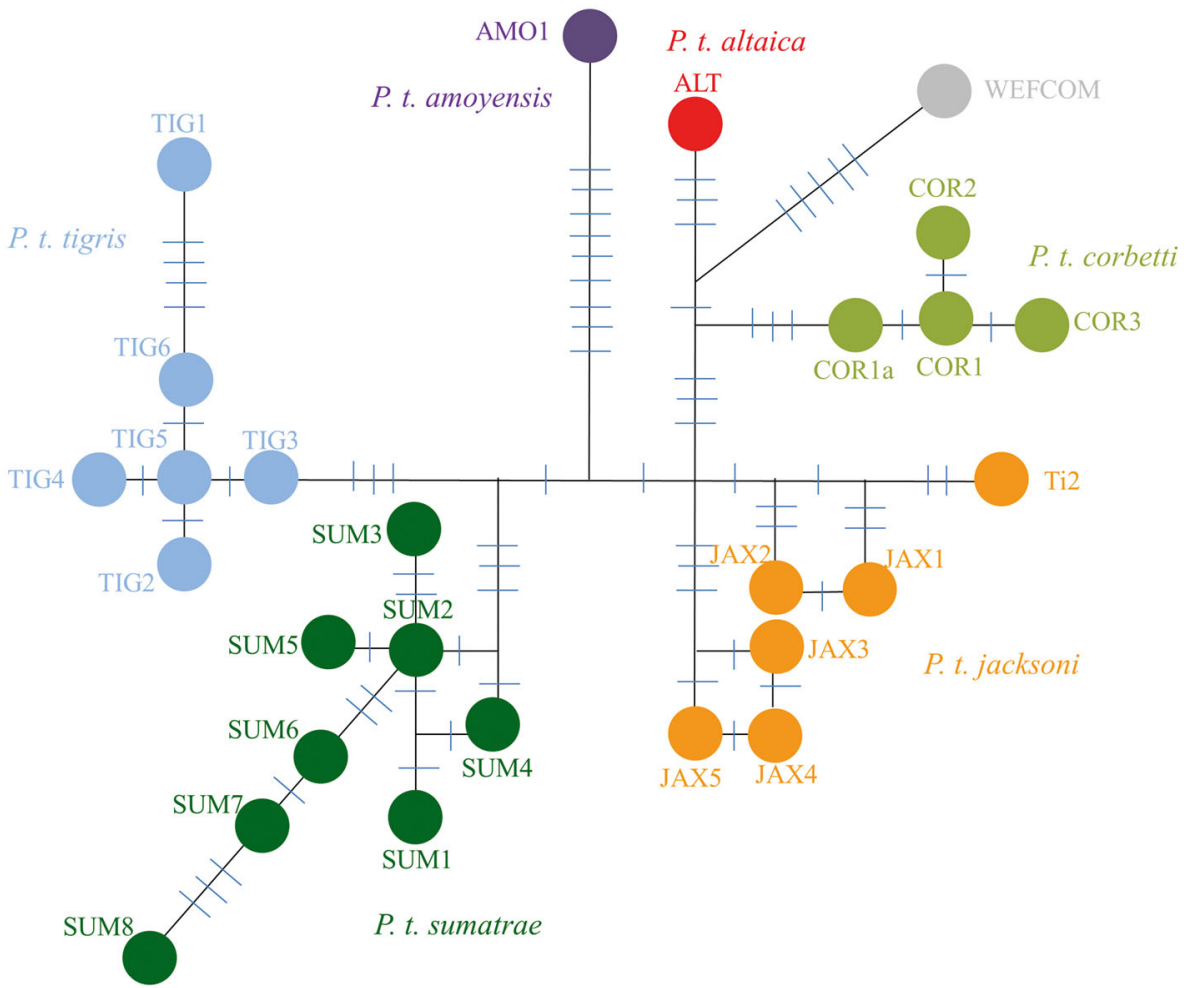

Fig. 1. Phylogeny of mtDNA haplotypes of extant tiger Panthera tigris subspecies based on 4078 bp of concatenated sequences following Luo et al. (2004). (A) Results from maximum parsimony (MP), neighbour-joining (NJ), maximum likelihood (ML) and Bayesian analyses were similar, so only the MP tree is shown here. The numbers above each branch represent the statistical support in percentages for the MP, NJ, ML, and Bayesian analyses, respectively. Dashes represent statistical support values $<50$. The numbers below each branch represent the number of changes. (B) The median-joining network demonstrates the evolutionary relationship between our 2 samples and previous reports (Luo et al. 2004). Each circle represents a haplotype, and the marks on each branch indicate mutation points. Circle size is not proportional to the haplotype frequency. Both the MP phylogenetic tree and the median-joining network support an additional Indochinese haplotype (WEFCOM), which evolved from a common ancestor between $P$. $t$. altaica and P. $t$. corbetti. The new Malayan haplotype (Ti2) descended from the same historic ancestor as P. t. jacksoni 
Indochina and Far Eastern regions of Asia, and substantial population genetic substructure within Indochinese tigers. The southern haplotype, Ti2, clustered with JAX1 and JAX2 haplotypes and formed a monophyletic group within the Malayan tiger subspecies $P$. $t$. jacksoni. These results were supported by the median-joining network analysis and suggested a complex phylogeographic pattern within $P$. $t$. corbetti in that the WEFCOM haplotype is different from other $P$. t. corbetti haplotypes, and that the Ti2 haplotype belongs to P. t. jacksoni (Fig. 1B).

The analysis of the longer mtDNA sequence showed that the WEFCOM haplotype appears to be more closely related to $P$. $t$. altaica than to other $P$. $t$. corbetti haplotypes with high statistical support in all algorithms. However, the number of changes from the WEFCOM haplotype to the haplogroup of $P$. $t$. altaica (15 nucleotides) is larger than that to the haplogroup of P. t. corbetti (13 nucleotides; Fig. 2). This result further indicates a close and complex relationship between the 2 subspecies. All analyses using the MP, ML and Bayesian approaches consistently supported the finding that the lineage of $P$. $t$. jacksoni diverged earlier than the splitting of $P$. $t$. corbetti and P. t. altaica.

\section{DISCUSSION}

Tiger populations with a sufficient number of breeding individuals to support the wider landscape are currently thought to occur in only 8 countries, including Thailand (Goodrich et al. 2015). Therefore, it is vitally important to understand the dynamics of the wild tiger population in Thailand. In the present study, we provide the first report of the complete mitogenome of the Malayan tiger and of novel haplotypes in the wild Indochinese tiger population in Thailand. We also demonstrate the phylogenetic relationship of these 2 subspecies to other tiger populations in Asia.

The results of our phylogenetic analyses are in agreement with the subspecies classification previously described (Luo et al. 2004), but indicate phylogenetic differences between the WEFCOM haplotype and other Panthera tigris corbetti haplotypes found across Asia. We found that the WEFCOM haplotype contained the 14711A SNP of P. t. altaica, a common SNP shared among $P$. t. altaica and $P$. $t$. corbetti (15756C), and no subspecies-specific SNPs described for $P$. $t$. corbetti (Luo et al. 2004). Our results suggest that the WEFCOM haplotype is

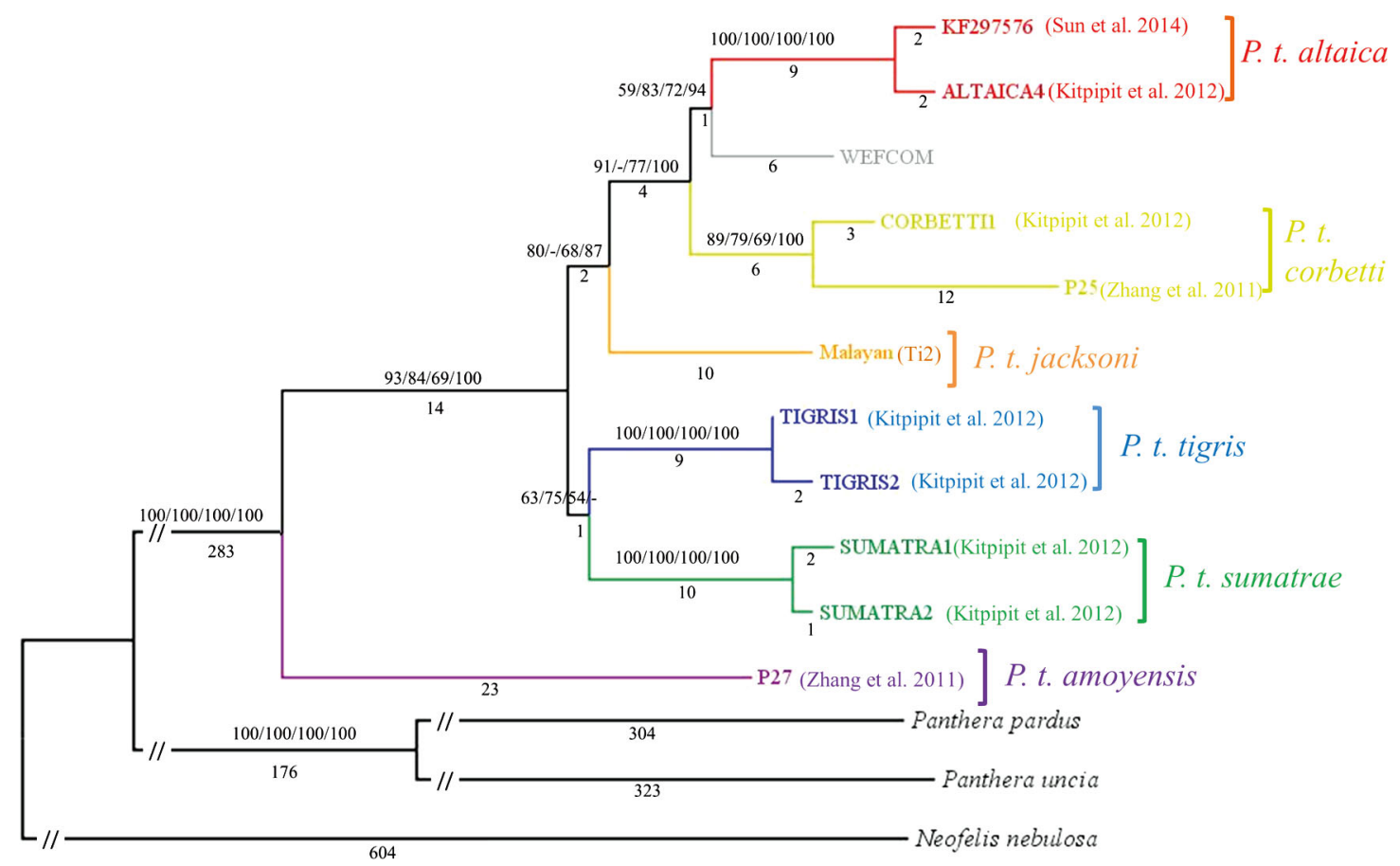

Fig. 2. Maximum parsimony (MP) phylogenetic tree based on 10140 bp of mitochondrial sequence (Zhang et al. 2011, Kitpipit et al. 2012, Sun et al. 2014) excluding the control region (CR) and the numt-contaminated fragments. The numbers above each branch represent the bootstrap values for the MP, neighbour-joining, maximum likelihood and Bayesian analyses, respectively. Dashes represent bootstrap values $<50$. The number below each branch represents the number of changes. The placement of the WEFCOM haplotype was consistent in all methods with high statistical support 
closely related to P. t. altaica and that there may be more complex genetic structure in Indochinese tigers than previously thought. As expected, the Ti2 haplotype sampled in southern Thailand formed a monophyletic group with JAX haplotypes and indicates that P. t. jacksoni diverged from a common Indochinese ancestor earlier than the divergence between P. t. corbetti and P. t. altaica.

A recent genetic study of tigers in the Sunda region suggests that the tiger subspecies now found in much of southeast Asia and the Russian Far East (P. t. corbetti, P. t. jacksoni and P. t. altaica) most likely evolved from a common ancestor in a second wave of expansion and divergence from $P . t$. amoyensis in China (Xue et al. 2015). Amur tigers (P. t. altaica) and Indochinese tigers $(P . t$. corbetti) have also been shown to be sister taxa separated by only a few mitochondrial steps. The close phylogenetic relationship between 1 wild P. t. altaica (GenBank KF297576) from China (Sun et al. 2015) and the WEFCOM individual in this study provides further support for a recent common ancestor for these 2 haplotypes, and hints at further local Indochinese substructure. Thus, tiger populations in northern Thailand may contain some of the haplotypes present in the ancestral population when tigers expanded out of China and into Russia and mainland Asia. Thailand is now the only country in the region with a reasonable population of P. $t$. corbetti tigers, as they have largely been poached from other neighbouring countries such as China, Laos and Cambodia. Thus, the existence of new haplotypes in Thailand also highlights its critical geographic position between the populations of $P$. $t$. altaica in the Russian Far East and P. t. jacksoni in Malaysia.

The discovery of 2 distinct sequences containing numt in this study is notable, as it suggests the presence of additional numt in multiple fragments, as has been reported in species such as the domestic cat, human and chimpanzee (Lopez et al. 1994, 1996, Tourmen et al. 2002, Antunes et al. 2007, HazkaniCovo \& Graur 2007). Therefore, future studies involving the complete mitochondrial genome of tigers must take care to check for confounding sequences between the 2 ends of F2 numt, which should be excluded from any downstream phylogenetic analysis.

\section{CONCLUSIONS}

Here, we have provided an account of the complete mitogenome of Panthera tigris corbetti and P. t. jacksoni, and have provided further information on the phylogenetic relationship between these 2 tiger subspecies in Thailand. Our results also provide evidence for the close relationship between $P$. $t$. altaica and $P$. $t$. corbetti, and support the importance of WEFCOM to the Asian tiger population.

Acknowledgements. We acknowledge the Graduate School, Kasetsart University, Thailand, for supporting this project. We are also grateful to all the project collaborators in the Zoological Park Organization under the Royal Patronage of His Majesty the King, the Department of National Parks, Wildlife and Plant Conservation and associated field researchers who provided the samples. We also thank Anyalak Wachirachaikarn and Phasit Charoenkwan for their advice on the bioinformatics analysis.

\section{LITERATURE CITED}

Antunes A, Pontius J, Ramos MJ, O'Brien SJ, Johnson WE (2007) Mitochondrial introgressions into the nuclear genome of the domestic cat. J Hered 98:414-420

Bandelt HJ, Forster P, Rohl A (1999) Median-joining networks for inferring intraspecific phylogenies. Mol Biol Evol 16:37-48

Carter MJ, Milton ID (1993) An inexpensive and simple method for DNA purifications on silica particles. Nucleic Acids Res 21:1044

Delisle I, Strobeck C (2002) Conserved primers for rapid sequencing of the complete mitochondrial genome from carnivores, applied to three species of bears. Mol Biol Evol 19:357-361

DNP (Department of National Parks, Wildlife and Plant Conservation) (2010) Thailand Tiger Action Plan 20102011. Ministry of Natural Resources and Environment Thailand, Bangkok

Driscoll CA, Yamaguchi N, Bar-Gal GK, Roca AL, Luo S, Macdonald DW, O'Brien SJ (2009) Mitochondrial phylogeography illuminates the origin of the extinct Caspian tiger and its relationship to the Amur tiger. PLoS One 4: e4125

Goodrich J, Lynam A, Miquelle D, Wibisono H and others (2015) Panthera tigris. The IUCN Red List of Threatened Species 2015: e.T15955A50659951. www.iucnredlist.org

Hall TA (1999) BioEdit: a user-friendly biological sequence alignment editor and analysis program for Windows 95/98/NT. Nucleic Acids Symp Ser 41:95-98

Hazkani-Covo E, Graur D (2007) A comparative analysis of numt evolution in human and chimpanzee. Mol Biol Evol 24:13-18

Kim JH, Antunes A, Luo SJ, Menninger J, Nash WG, O'Brien SJ, Johnson WE (2006) Evolutionary analysis of a large mtDNA translocation (numt) into the nuclear genome of the Panthera genus species. Gene 366: 292-302

Kitpipit T, Tobe SS, Linacre A (2012) The complete mitochondrial genome analysis of the tiger (Panthera tigris). Mol Biol Rep 39:5745-5754

> Lopez JV, Yuhki N, Masuda R, Modi W, O'Brien SJ (1994) Numt, a recent transfer and tandem amplification of mitochondrial DNA to the nuclear genome of the domestic cat. J Mol Evol 39:174-190 
Lopez JV, Cevario S, O'Brien SJ (1996) Complete nucleotide sequences of the domestic cat (Felis catus) mitochondrial genome and a transposed mtDNA tandem repeat (Numt) in the nuclear genome. Genomics 33:229-246

Luo SJ, Kim JH, Johnson WE, van der Walt J and others (2004) Phylogeography and genetic ancestry of tigers (Panthera tigris). PLoS Biol 2:e442

Maźak V (1981) Panthera tigris. Mamm Species 152:1-8

Mondol S, Bruford MW, Ramakrishnan U (2013) Demographic loss, genetic structure and the conservation implications for Indian tigers. Proc R Soc Lond B Biol Sci 280:20130496

Posada D (2008) jModelTest: phylogenetic model averaging. Mol Biol Evol 25:1253-1256

Ronquist F, Huelsenbeck JP (2003) MrBayes 3: Bayesian phylogenetic inference under mixed models. Bioinformatics 19:1572-1574

Ronquist F, Teslenko M, van der Mark P, Ayres DL and others (2012) MrBayes 3.2: efficient Bayesian phylogenetic inference and model choice across a large model space. Syst Biol 61:539-542

Simcharoen S, Pattanavibool A, Karanth KU, Nichols JD, Kumar NS (2007) How many tigers Panthera tigris are

Editorial responsibility: Mike Bruford, Cardiff, UK there in Huai Kha Khaeng Wildlife Sanctuary, Thailand? An estimate using photographic capture-recapture sampling. Oryx 41:447-453

> Sun Y, Lu T, Sun Z, Guan W and others (2015) Complete mitochondrial genome of a wild Siberian tiger. Mitochondrial DNA 26:663-664

Swofford DL (2002) Phylogenetic analysis using parsimony ( ${ }^{*}$ and other methods). Version 4. Sinauer Associates, Sunderland, MA

Tourmen Y, Baris O, Dessen P, Jacques C, Malthiery Y, Reynier P (2002) Structure and chromosomal distribution of human mitochondrial pseudogenes. Genomics 80: 71-77

Walston J, Robinson JG, Bennett EL, Breitenmoser U and others (2010) Bringing the tiger back from the brinkthe six percent solution. PLoS Biol 8:e1000485

> Xue HR, Yamaguchi N, Driscoll CA, Han Y and others (2015) Genetic ancestry of the extinct Javan and Bali tigers. J Hered 106:247-257

> Zhang W, Yue B, Wang X, Zhang X and others (2011) Analysis of variable sites between two complete South China tiger (Panthera tigris amoyensis) mitochondrial genomes. Mol Biol Rep 38:4257-4264

Submitted: December 11, 2014; Accepted: March 17, 2016 Proofs received from author(s): April 25, 2016 